\title{
Patientenverfügung und Zwangsernährung
}

\author{
Anders als die Juristen Müller und Jenni in einem vorangegangenen SÄZ-Beitrag \\ kritisiert der Autor die Durchführung einer Zwangsernährung als schweren Eingriff \\ in die Grundrechte. Dies im Besonderen dann, wenn sie gegen den zuvor geäusser- \\ ten Willen des Betroffenen erfolgt. Eine Patientenverfügung dürfe nicht gering- \\ geschätzt werden.
}

«Niemand hat das Recht zu gehorchen.»

(Hannah Arendt)

Der Hungerstreik des zu einer mehrjährigen Freiheitsstrafe verurteilten Hanfbauern B. Rappaz und der in dieser Sache ergangene Bundesgerichtsentscheid [1] haben in der Schweizerischen Ärztezeitung (SÄZ) zu einer lebhaften Diskussion um die Anwendung von medizinischen Zwangsbehandlungen bei Personen im Strafvollzug geführt [2-9]. Kritisiert wurde der Entscheid des Bundesgerichtes, trotz vorhandener Patientenverfügung und fehlender gesetzlicher Grundlage mittels Rückgriff auf die sog. Polizeigeneralklausel eine Zwangsernährung als verhältnismässigen Eingriff zu befürworten, um eine Fortdauer der Haft zu gewährleisten. In der SÄZ 8/2011 äusserten sich zwei Juristen der Universität Bern zur umrissenen Thematik [10]. Ihre provokanten Aussagen fordern zu einer Entgegnung heraus, wobei das Schwergewicht auf die Bedeutung von Patientenverfügungen gelegt wird.

\section{Einleitung}

Das Recht auf persönliche Freiheit sowie auf seelische und körperliche Integrität ist als Grundrecht in der Bundesverfassung verankert (Art. $10 \quad \mathrm{BV}^{1}$ ). Das Schweizerische Zivilgesetz regelt den äusseren Schutz der Persönlichkeit, indem prinzipiell jede Verletzung als widerrechtlich gilt, sofern sie nicht durch Einwilligung der betroffenen Person, durch ein überwiegendes privates oder öffentliches Interesse oder durch ein Gesetz gerechtfertigt ist (Art. $28 \mathrm{ZGB}^{2}$ ). Dabei kommt der Einwilligung die höchste Bedeutung zu. In analoger Weise wie das Grundrecht auf Integrität werden die Glaubens- und Gewissensfreiheit verfassungsmässig geschützt (Art. 15 BV). Dieser Schutz beinhaltet durchaus das Recht des Urteilsfähigen, eine medizinische Behandlung oder eine zwangsweise Ernährung abzulehnen - möge die Massnahme nach herrschender Lehre noch so indiziert sein und die persönliche Entscheidung auf die Umgebung noch so «unvernünftig» wirken. Bei einer Zuwiderhandlung gegen die persönliche Integrität kann der Betroffene das Gericht anrufen, um eine Verletzung feststellen, verbieten oder beseitigen zu lassen (Art. 28 f. ZGB). Das Strafgesetzbuch kennt Tatbestände der vorsätz-

\section{Directives anticipées et alimenta-} tion forcée

La longue grève de la faim suivie par B. Rappaz alors qu'il était condamné à une peine de prison et l'alimentation forcée de ce dernier ordonnée au sommet de I'Etat ont provoqué des discussions controversées. L'arrêt du Tribunal fédéral (6B_599/2010) et le point de vue juridique de $M$. Müller et $C$. Jenni présenté dans un article du BMS $\left(n^{\circ} 8 / 2011\right)$ soulèvent des questions pressantes autour des mesures médicales imposées. L'auteur porte un regard critique sur ledit article et nous éclaire notamment sur la valeur des directives anticipées, entre autres aussi à la lumière du nouveau droit de la protection de l'adulte.

lichen und fahrlässigen Körperverletzung (Art. 122, 123, $125 \mathrm{StGB}^{3}$ ), wobei die Tat - je nach Schweregrad der Tatbestandsumschreibung - von Amts wegen oder auf Antrag der geschädigten Person verfolgt wird. Grundsätzlich stellt jeder ärztliche Heileingriff eine Körperverletzung dar, die erst durch die Einwilligung des urteilsfähigen, hinreichend aufgeklärten Patienten gerechtfertigt wird. Verfassungsmässig verankert ist auch das sog. Diskriminierungs- und Willkürverbot (Art. 8 und 9 BV), wonach niemand - auch ein Strafgefangener nicht - aufgrund innerer oder äusserer Merkmale benachteiligt werden darf und jede Person den Anspruch hat, von staatlichen Organen ohne Willkür behandelt zu werden. Ferner bedarf die Einschränkung von Grundrechten einer gesetzlichen Grundlage, ausgenommen sind gemäss Art. 36 BV Fälle einer ernsten, unmittelbaren und nicht anders abwendbaren Gefahr (sog. polizeiliche Generalklausel). Grundrechtseinschränkungen müssen zudem stets verhältnismässig sein und durch ein öffentliches Interesse oder durch den Schutz von Grundrechten Dritter gerechtfertigt sein. 


\section{Zum Artikel von Müller und Jenni in der Schweizerischen Ärztezeitung}

In ihrem Artikel vertreten die beiden Autoren sinngemäss die Auffassung, dass der Staat im Falle eines lebensbedrohlichen Hungerstreiks eines Strafgefangenen als ultima ratio zu einer einmaligen Zwangsernährung schreiten sollte. Die staatliche Fürsorgepflicht zum Schutz des Lebens überwiege dabei den Schutz des Wunsches auf ein selbstbestimmtes Sterben. Dies sei umso mehr der Fall, als «ohnehin Zweifel» an dem zu einem früheren Zeitpunkt geäusserten Sterbewillen bestünden. In einer Güterabwägung, die allen Aspekten Rechung trage, werde sich die Zwangsernährung tendenziell als eine verhältnismässige Massnahme erweisen, um den Gefangenen in einen psychischen und physischen Zustand zurückzuversetzen, der ihm erlaube, seine Situation bei vollem Bewusstsein zu überdenken. Wenn er sich jedoch nach der einmaligen Zwangsmassnahme für die Wiederaufnahme des Hungerstreiks entscheide, ende der mehrter Zuwendung in Form von Gesprächen, ärztlicher und pflegerischer Betreuung, Spitaleinweisung, Überwachung usw., also zu einer Sonderbehandlung, die durchaus attraktiv erscheinen kann.

\section{«Zurechnungsfähigkeit»}

Unter der Überschrift «Patientenverfügung» verwenden die Autoren überraschenderweise den im Strafrecht gebräuchlichen Begriff der Zurechnungsfähigkeit. Mit der Bestimmung der Zurechnungsfähigkeit wurde bis vor kurzem (Art. 10 und 11 StGB, alte Version) beschrieben, ob und in welchem Ausmass ein Beschuldigter zum Zeitpunkt einer Straftat in der Lage war, das Unrecht seiner Tat einzusehen und sein Handeln nach dieser Einsicht zu steuern. Mit Inkrafttreten der neuen StGB-Version ist der Begriff der Zurechnungsfähigkeiten durch den Terminus der Schuldfähigkeit abgelöst worden (Art. 19 StGB), prinzipiell lassen sich die beiden Bezeichnungen aber synonym verwenden.

\section{Es ist nicht ersichtlich, warum eine Zwangsernährung Nachahmer abschrecken sollte.}

staatliche Schutz- und Fürsorgeauftrag. Verweigere der mit der Zwangsernährung beauftragte Arzt den Dienstbefehl der übergeordneten Stelle, drohten ihm strafrechtliche Folgen, beispielsweise im Sinne der Körperverletzung durch Unterlassen (Art. 123 Ziff. 1, Art. 11 StGB), der unterlassenen Nothilfe (Art. 128 StGB), des Ungehorsams gegen amtliche Verfügungen (Art. 292 StGB) oder - im Todesfall - der Tötung infolge Unterlassung (Art. 111, Art. 11 StGB bzw. Art. 117, Art. 11 StGB) [10].

\section{Öffentliches Interesse}

Die Autoren weisen zunächst darauf hin, dass in den meisten Kantonen eine formell-gesetzliche Vorschrift fehle, die einen derart schweren Grundrechtseingriff wie eine Zwangsernährung legitimieren könne. Folglich müsse sich die Vollzugsbehörde in den meisten Kantonen auf die Polizeigeneralklausel berufen. Ein zur Rechtfertigung erforderliches öffentliches Interesse an einer Zwangsernährung sei problemlos zu finden, beispielsweise die Glaubwürdigkeit der Justiz, die Gleichbehandlung von Strafgefangenen oder die Verhinderung von Nachahmern. Diese etwas salopp hingeworfen erscheinende Liste - frei nach dem Motto «Wer den Hund schlagen will, findet schon den Stock» - erscheint nicht a priori plausibel, jedenfalls übersehen die Autoren, dass sich mit einzelnen Punkten genauso gut auch gegenteilig argumentieren lässt. Es ist beispielsweise nicht ersichtlich, warum eine (medienwirksame) Zwangsernährung Nachahmer abschrecken sollte. Intuition und Lerntheorie suggerieren eher das Gegenteil: Ein Hungerstreik führt zu ver-
Bei der Frage nach der Validität von Patientenverfügungen spricht man üblicherweise von Urteilsfähigkeit und meint damit die Fähigkeit eines Menschen, vernunftgemäss zu handeln, sofern diese Fähigkeit nicht beispielsweise durch eine Geisteskrankheit, eine Geistesschwäche oder ähnliche Zustände beeinträchtigt wird (Art. 16 ZGB). Eine intakte Urteilsfähigkeit beinhaltet die Fähigkeit, im Hinblick auf eine anstehende Entscheidung - im vorliegenden Fall das Erstellen einer Patientenverfügung - Informationen zu verstehen, Konsequenzen aus verschiedenen Varianten gegeneinander abzuwägen, die Informationen im Kontext des eigenen Wertsystems zu gewichten und schliesslich das Vermögen, eine Wahl zu treffen und den Entscheid zu vertreten [11].

\section{Wert einer Patientenverfügung}

Die beiden Autoren offenbaren in ihrem Artikel eine bemerkenswerte Geringschätzung gegenüber dem Instrument der Patientenverfügung. Sie argumentieren in einem ersten Schritt, dass im Kontext eines Hungerstreiks ein Todeswunsch nicht sicher vorliege, da der Gefangene vielmehr in besseren Umständen leben wolle und lediglich ein gewisses Sterberisiko in Kauf nehme; aus diesem Grund sei der Geltungsanspruch einer Patientenverfügung zu relativieren.

Diese Argumentation überzeugt nicht. Würde man ihr folgen, wäre wohl die grosse Mehrzahl der Patientenverfügungen Makulatur. Die wenigsten Menschen, die eine Patientenverfügung erstellen, sehnen den Tod herbei - im Gegenteil, sie möchten leben. Sie bezeichnen aber unterschiedlich weit im Voraus Krank- 


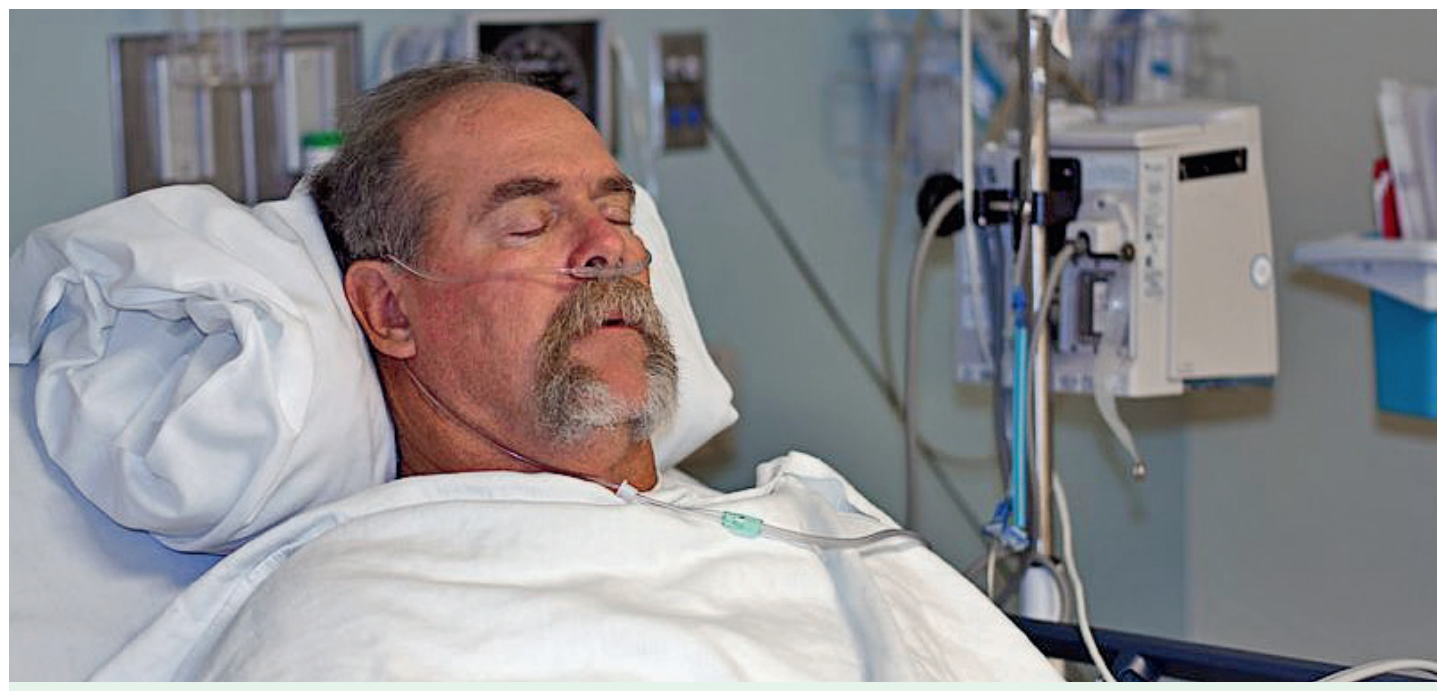

Wann ist bei einem Patienten die Urteilsfähigkeit über medizinische Massnahmen noch bzw. wieder gegeben?

heits- bzw. Leidenszustände, in denen sie bestimmte therapeutische Interventionen dereinst akzeptieren oder ablehnen. Wenn sie sich dabei beispielsweise gegen lebenserhaltende medizinische Behandlungen aussprechen, tun sie dies unter Inkaufnahme des Todes. Die Autoren widersprechen implizit ihrer eigenen Argumentation, indem sie lediglich einmalig eine staatliche Zwangsernährung fordern und anschliessend bereit sind, eine unmittelbar nach der Zwangsmassnahme geäusserte Bekräftigung der ursprünglichen Verfügung zu akzeptieren. Allerdings bleiben sie die Erklärung schuldig, warum die Willensbekundung einer nach langer Nahrungskarenz geschwächten, durch Zwangsernährung eben gerade auf ein im Laufe des Hungerstreikes aber kontinuierlich bis zur «Unzurechnungsfähigkeit» dahinschwinde. Nach Erreichen dieses Zustandes dauere es mitunter mehrere Wochen bis zum Eintritt der Bewusstlosigkeit. In dieser Periode könne ein früher gefasster Wunsch nicht mehr adäquat überdacht und ggf. widerrufen werden. Zudem würden aufgestaute Emotionen und die Angst vor einem Gesichtsverlust vom «vernebelten Restverstand» in dieser Phase überbewertet, was einem Streikabbruch aus freien Stücken entgegenstehe. Vor diesem Hintergrund könne nicht mehr von einer «klaren Willenskundgabe» und damit auch nicht mehr von einer «gültigen Patientenverfügung» gesprochen werden.

\section{Die Autoren offenbaren in ihrem Artikel eine bemerkenswerte Gering- schätzung gegenüber dem Instrument der Patientenverfügung.}

gewisses Minimalgewicht gebrachten Person valider sein soll als eine früher geäusserte. Die vorgeschlagene Massnahme der einmaligen Zwangsernährung erscheint daher willkürlich, genauso gut könnten zwei, drei oder noch mehr Zwangszyklen empfohlen werden, bis einer Patientenverfügung endlich ihre Gültigkeit zugestanden wird. Analog liesse sich die einmalige intraoperative Bluttransfusion bei einem Zeugen Jehovas trotz expliziter Ablehnung rechtfertigen mit der Begründung, er könne sich ja danach noch einmal dazu äussern, beim nächsten Mal werde man seinem Wunsch dann entsprechen.

\section{Zweck einer Patientenverfügung}

In einem zweiten Schritt führen die Autoren aus, dass «die Zurechnungsfähigkeit» - gemeint ist wohl die Urteilsfähigkeit (s. oben) - zu Beginn der Nahrungsverweigerung zwar noch vorhanden sein möge, diese
Auch dieses Glied der Argumentationskette vermag nicht zu überzeugen, da ihm ein grobes Missverständnis zum Wesen der Patientenverfügung zugrunde liegt. Die Autoren messen der erlöschenden Geisteskraft im Rahmen der prolongierten Nahrungsverweigerung und später der eingetretenen Bewusstlosigkeit eine überragende Bedeutung bei; die zu Beginn des Hungerstreiks vorhandene Urteilsfähigkeit ist für sie dagegen weitgehend wertlos. Würde man dieser Sichtweise folgen, käme kaum je eine Patientenverfügung auf Anhieb zum Tragen. Sobald nämlich eine Aufhebung der Urteilsfähigkeit einträte, müsste die Verfügung mit Verweis auf eben diesen aktuell «vernebelten Restverstand» bzw. die eingetretene Urteilsunfähigkeit für ungültig erklärt werden ein Zirkelschluss ohne Entrinnen. Ein Ausweg würde sich laut Autoren durch das Eintreten eines identischen Zweitereignisses eröffnen, da erst dann (wie im 
konkreten Fall) die Fürsorge- und Schutzpflicht des Staates - und damit nach Überzeugung der Autoren die Pflicht der Ärzte, Zwang auszuüben - erlösche. Diese Sichtweise unterminiert den einzigen Zweck jeder Patientenverfügung, nämlich die Vorwegnahme eines Zustandes, in der die betroffene Person eben gerade mangels Urteilsfähigkeit nicht mehr selber entscheiden kann, welchen medizinischen Massnahmen sie zustimmt und welchen nicht [12].

\section{Patientenverfügung und die SAMW}

Ironischerweise befinden sich die Autoren mit ihrer Relativierung der Patientenverfügung auf einer früheren Linie der Schweizerischen Akademie der Medizinischen Wissenschaften SAMW, deren Guidelines laut Müller und Jenni im Geltungsbereich des öffentlichen Rechts ohnehin nichts zu suchen haben, da es sich um rein private Standesregeln handle, welche die Ärzteschaft überdies im Sinne einer Machtfrage zu instrumentalisieren trachte. In der aus dem Jahr 2005 stammenden medizinisch-ethischen Leitlinie «Recht der Patientinnen und Patienten auf Selbstbestimmung» [11] vertrat die SAMW die inzwischen revidierte Position, wonach eine Patientenverfügung lediglich ein gewichtiges Indiz bei der Ermittlung des Patientenwillens darstelle. Da die Patientenverfügung so die damalige Argumentation - eine Vorausverfügung für kaum vorhersehbare existenzielle Situationen darstelle, könne sie weder rechtlich noch ethisch mit einer Jetzt-für-jetzt-Erklärung des Urteilsfähigen verglichen werden. Zudem werde grundsätzlich eine Einwilligung zu einer Behandlung nur nach hinreichender Aufklärung wirksam, was bei Patientenverfügungen häufig nicht der Fall sei, da diese oft sogar ohne jede Aufklärung errichtet würden [11].

\section{Patientenverfügung und neues \\ Erwachsenenschutzrecht}

Das neue Erwachsenenschutzrecht (nZGB $)$, das voraussichtlich 2013 in Kraft tritt und erstmals auf eidgenössischer Ebene den Umgang mit Patientenverfügungen regelt (Art. 370-373 nZGB), geht weiter und verpflichtet die Ärzte, einer Vorausverfügung grundsätzlich zu entsprechen. Das Gesetz geht davon aus, dass die verfügende Person sich im Besitz der zur Willensbildung nötigen Informationen befindet und auf eine zusätzliche Aufklärung verzichtet. Nach neuem Gesetz gibt es nur drei Situationen, in denen von einer Patientenverfügung abgewichen werden darf: bei einem Verstoss gegen gesetzliche Vorschriften sowie bei begründetem Zweifel an der Willensfreiheit des Verfügenden oder daran, ob die Anordnung noch dem aktuellen mutmasslichen Willen der Person entspricht. In den beiden letzten Fällen müssen aber konkrete Hinweise vorliegen; ein Hinterfragen jedes Mal, wenn die Verfügung eine den Ärzten (oder den Justizvollzugsbehörden) unpassend erscheinende Lösung vorschlägt, ist nicht vorgesehen [12]. Die SAMW hat schnell reagiert und diese neuen Bestimmungen kurz nach der Gutheissung des Gesetzes durch die eidgenössischen
Räte bereits 2009 in ihre medizinisch-ethischen Leitlinie «Patientenverfügungen» aufgenommen [13].

\section{Fazit}

Die beiden Autoren verlangen eine Art blinden Gehorsams, wenn sie schreiben, dass es einen Gefängnisarzt letztlich nicht zu interessieren braucht, ob ein Richterspruch klug, überzeugend oder gar medizinethisch richtig ist. Mit anderen Worten: Es braucht ihn auch dann nicht zu interessieren, wenn der Richterspruch unklug, nicht überzeugend oder medizinethisch falsch ist - er muss den Befehl ausführen oder den Dienst quittieren. Falls er sich weigert, drohen ihm disziplinar- oder gar strafrechtliche Konsequenzen. Über ihrer problematischen Argumentation vergessen die Autoren, dass dem Gefängnisarzt weitere Ungemach droht: vonseiten des Patienten, der angesichts der offensichtlichen Missachtung einer Vorausverfügung im strafrechtlichen Sinne eine vorsätzliche Körperverletzung geltend machen kann. Angesichts der Drohkulisse ist es beruhigend zu wissen, dass Ärzte es wagen, sich ihres Verstandes zu bedienen.

\section{Literatur}

1 BGE 136 IV 97 (Urteil 6B 599/2010 vom 26. August 2010). www.bger.ch/index/juridiction/jurisdiction-inherit-template/jurisdiction-recht/jurisdiction-rechtleitentscheide1954-direct.htm

2 Carasso B. Le cas Bernard Rappaz. Schweiz Ärztezeitung. 2010;91(32):1184.

3 De Haller J. Die Medizin, die Ärzte und die Richter ... Schweiz Ärztezeitung. 2010;91(39):1509.

4 De Haller J, Théraulaz P, Suter P, Kind C, Gravier B, Schneider A et al. Hungerstreik im Gefängnis. Schweiz Ärztezeitung. 2010;91(39):1518-20.

5 Gravier B, Wolff H, Sprumont D, Ricou B, Kind C, Eytan A et al. Ein Hungerstreik ist eine Protesthandlung. Schweiz Ärztezeitung. 2010;91(39):1521-5.

6 Bouvier B. Brief des Internationalen Komitees vom Roten Kreuz IKRK an den Präsidenten der FMH. Schweiz Ärztezeitung. 2010;91(39):1526.

7 Geiser M. Brief an die Redaktion der Schweizerischen Ärztezeitung. Schweiz Ärztezeitung. 2010; 91(39):1526.

8 Clodius L. Zwangsernährung. Schweiz Ärztezeitung. 2010;91(43):1690.

9 Bättig J. Hungerstreik und Zwangsernährung. Schweiz Ärztezeitung. 2011;92(10):365.

10 Müller M, Jenni C. Hungerstreik und Zwangsernährung. Schweiz Ärztezeitung. 2011;92(8):284-7.

11 Recht der Patientinnen und Patienten auf Selbstbestimmung. Medizinisch-ethische Grundsätze der SAMW. 24. November 2005. www.samw.ch/de/Ethik/ Richtlinien/Aktuell-gueltige-Richtlinien.html

12 Botschaft des Bundesrates zur Änderung des Schweizerischen Zivilgesetzbuches (Erwachsenenschutz, Personenrecht und Kindesrecht) vom 28. Juni 2006.

13 Patientenverfügungen. Medizinisch-ethische Richtlinien und Empfehlungen der SAMW. 19. Mai 2009. www.samw.ch/de/Ethik/Richtlinien/Aktuellgueltige-Richtlinien.html 